\title{
Creare Valore Sanitario: nuove prospettive per il management delle aziende sanitarie
}

\begin{abstract}
The value creation is the primary target of a firm and to this pattern is inspired each managerial instrument, behaviour and action.

For particular firms as health companies, the pattern of value creation can be well - grounded, provided that it takes care of their particular attributes. Therefore this article is structured on the following methodology of analysis: first of all the pattern of value creation for private firms is shown making clear the meaning of value creation compared with firm's strategic targets. Then the particular attributes of health companies and the meaning that value creation can undertake in these circumstances are analysed. If the creation of health value can become the strategic target of a health company, it is essential a valuation of the "key variables" marking out the way to create value. At last the change of classical instruments of management and administration control are described.

To create health value is the real strategic target of health companies. After years of prevailing or exclusive orientation to efficiency, now managerial instruments rediscover the efficacy and the quality of medical treatments as "key variables" to program and control.

Old management tools, such as the budget or the reporting, can become an excellent support for every operator, first of all the medical class that, because of various motives, was (and is) particularly refractory to these instruments.

The pattern of value creation can become the philosophy which has to inspire all the health management instruments, and the common language for all the cultures and the professions inside the health companies. Concluding, the pattern of value creation can be an interesting proposal to innovate control systems of health companies and to put an end to the impasse of most of the health management's applications, but, it required a new co-operation between managers and medical class. Only if the medical class will accept valuation and measurement systems of activity, the value creation will stop to be just a theory and will become a reality.
\end{abstract}

Farmeconomia e percorsi terapeutici 2000; 1 (1): 25-34

\section{INTRODUZIONE}

La sanità italiana è stata interessata in questi ultimi anni da notevoli spinte all'introduzione di modelli manageriali.

Se facessimo il punto della situazione a distanza di alcuni anni dall'avvio del processo di cambiamento avremo un quadro composto da luci e da ombre.

Le luci consistono indubbiamente nel fatto che sempre più si sente parlare di controllo di gestione, di budget, di reporting e così via, e che dopo tante parole inizino a seguire le prime applicazioni concrete di tali strumenti.

A queste luci, bisogna però aggiungere una serie consistente di zone d'ombra; facciamo riferimento in particolare al fatto che:

? i nuovi strumenti di management e di controllo di gestione sono stati interpretati prevalentemente come strumento per ridurre e tagliare i costi;
? Scarsa attenzione è stata posta dai modelli di management all'efficacia e alla qualità delle cure;

? come conseguenza di questo, la componente medica, pur dovendo rivestire un ruolo primario nell'applicazione e utilizzo di tali strumenti, ha finito per essere in molti casi la grande esclusa.

I limiti appena citati costituiscono senz' altro un valido stimolo per riflettere e ripensare i modelli e gli strumenti di management sanitario.

In questa prospettiva, il presente articolo ha l'obiettivo di proporre un approccio innovativo al management sanitario basato sul modello della creazione di valore sanitario.

Il modello in questione cerca di ridare centralità, nella filosofia, nelle logiche e negli strumenti operativi del management, alle variabili spesso trascurate, quali l'efficacia e la a Università di Torino; Nagima Divisione Sanità Torino

Corrispondenza a: Dott. Salvatore Nieddu o Prof. Giorgio Donna Tel.011.630.08.11 E-mail:nagima@tin.it 


\section{Nota $\mathbf{i}$}

Remunerazioni che assumeranno diverse configurazioni: prezzi di acquisto per le risorse messe a disposizione dai fornitori (materie prime, macchinari, servizi ecc.); salari, stipendi per il personale; tassi di interesse per $i$ finanziatori esterni; rendimenti, sotto forma di reddito o di incremento del capitale, per gli azionisti. qualità della cura che costituiscono il vero obiettivo di un'azienda sanitaria.

\section{MATERIALI E METODI}

Creare valore è il primario obiettivo di un'impresa e a tale modello si ispirano gli strumenti manageriali, i comportamenti e le azioni di ogni operatore.

Per particolari imprese come le aziende sanitarie, il modello della creazione di valore può essere valido a patto che venga declinato alla luce delle peculiarità che le caratterizzano.

$\mathrm{Su}$ questa logica l'articolo si struttura secondo la seguente metodologia di analisi.

Dapprima è illustrato il modello di creazione di valore per le imprese private mettendo a fuoco il significato di creazione di valore rispetto agli obiettivi strategici d'impresa.

Successivamente si analizzano le peculiarità delle aziende sanitarie e il significato che la creazione di valore può assumere in queste particolari realtà.

Se creare valore sanitario può diventare l'obiettivo strategico di un'azienda sanitaria risulta fondamentale, a questo punto, valutare quali sono le variabili chiave da cui questo obiettivo dipende e quali risultano essere pertanto i percorsi da seguire per creare valore.

L'ultima parte del lavoro è dedicata ad analizzare come cambiano, alla luce di questa nuova impostazione, i classici strumenti di management e di controllo di gestione, e come questi ultimi possano costituire un supporto di tutti gli operatori per finalizzare la propria azione a questo fondamentale obiettivo.

\section{RISULTATI}

Creare valore sanitario è il vero obiettivo strategico delle aziende sanitarie.

Dopo anni di prevalente o esclusivo orientamento all'efficienza e al breve periodo gli strumenti manageriali riscoprono l'efficacia, la qualità e l'appropriatezza delle cure, come variabili chiave da programmare e da controllare.

I vecchi "attrezzi" del management quali il budget e il reporting possono in quest'ottica arricchirsi e costituire un ottimo supporto per tutti gli operatori, in primo luogo per la classe medica, che per vari motivi risultava (e risulta) essere più riluttante e meno coinvolta nell'utilizzo di tali strumenti.

Il modello della creazione di valore può diventare la filosofia di fondo cui ispirare tutti gli strumenti del management sanitario e può costituire la lingua comune per facilitare l'incontro, il dialogo e il confronto delle diverse professionalità e culture che convivono all'interno delle aziende sanitarie.

\section{DISCUSSIONE}

La creazione di valore come obiettivo strategico d'impresa

L'obiettivo strategico di un'impresa privata è quello di creare valore.

Creare valore significa, in buona sostanza, fare qualcosa che "valga la pena", ovvero essere in grado di soddisfare tutti i portatori di interessi dell'impresa:

? i portatori di risorse, in via prioritaria gli azionisti, ma anche il personale, $i$ fornitori, $i$ finanziatori esterni, attraverso adeguate remunerazioni dei loro sforzi; ${ }^{i}$

? i portatori di bisogni, ovvero i clienti, la cui soddisfazione è misurata monetariamente dalla somma dei prezzi (i ricavi) che sono disposti a pagare per acquistare i beni o i servizi dell'impresa.

La creazione di valore rappresenta quindi l'imperativo e il parametro guida del management. Tale valore sintetizza sia la dimensione dell'efficacia sia quella dell'efficienza della gestione; creare valore è infatti indice di:

? efficacia, in quanto esprime la capacità dell'impresa di raggiungere l'obiettivo di fon-
Figura 1

Il "mestiere" dell'impresa

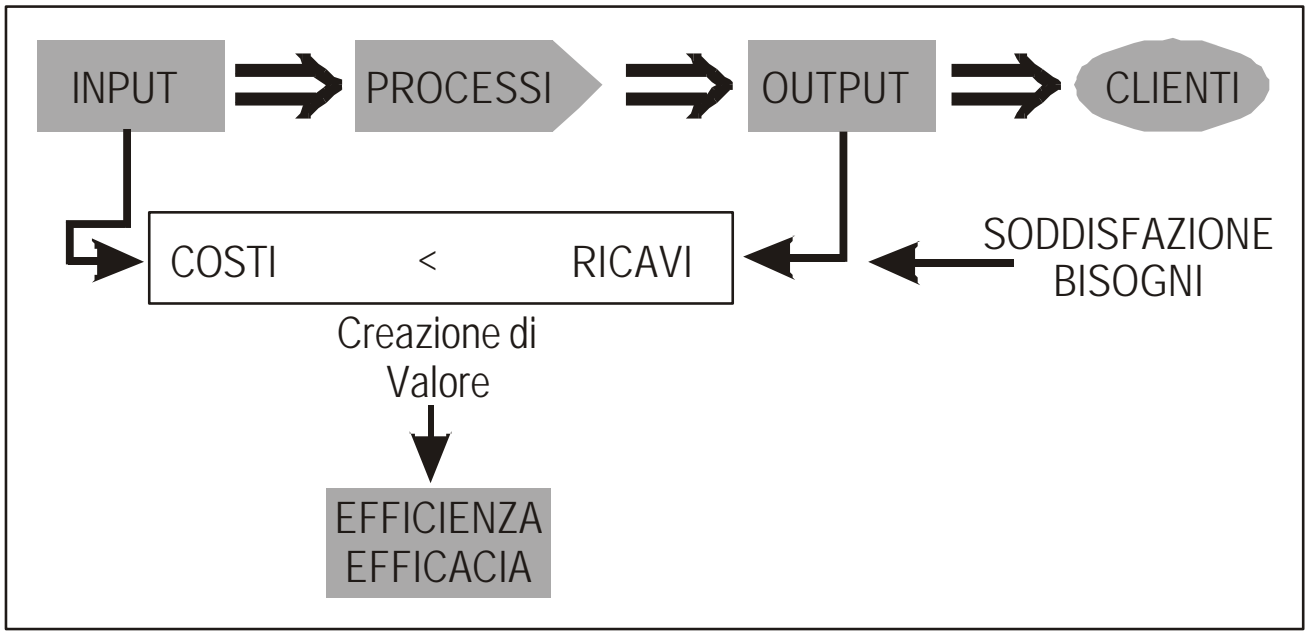


do, cioé il soddisfacimento dei portatori di interessi;

? efficienza, in quanto implica il razionale utilizzo delle risorse economiche.

Diverse sono le metodologie per valutare se un'impresa crea valore, ma tutte sono imperniate su misure di carattere economicofinanziario: per citarne una su tutte possiamo parlare del profitto economico quale differenza tra i ricavi e i costi sostenuti per la remunerazione di tutte le risorse acquisite dall'impresa ivi compreso il capitale degli azionisti.

Sebbene il concetto di profitto economico unitamente alle strumentazioni correlate per $\mathrm{i}$ calcoli economico-finanziari siano da tempo patrimonio della teoria economica, di recente, anche sull'onda della crescente competizione e concorrenza che sta caratterizzando i mercati, il razionale utilizzo delle risorse e l'ottimale remunerazione del capitale stanno assumendo una nuova centralità e rilevanza.

Tale fermento ha innescato a catena una serie di iniziative di revisione e rinnovamento degli strumenti di management tipicamente impiegati nelle imprese.

Queste iniziative si sono tradotte nell'introduzione di nuove metodologie o nella rivisitazione di quelle esistenti, per dare alla creazione di valore e alle variabili da cui questa dipende una forte centralità in tutti i momenti tradizionali dell'attività di programmazione e controllo della gestione: dalla formulazione delle strategie alla loro misurazione e controllo, dai sistemi di budget a quelli di reporting, dagli strumenti di supporto alla presa di decisioni sino ai meccanismi di incentivazione del personale.

Ad oggi si può pertanto dire che molte imprese stanno concretamente impiegando tali strumenti di gestione e che la creazione di valore è il vero faro guida del management delle imprese private.
La creazione di valore per le aziende sanitarie

Anche per imprese particolari come le aziende sanitarie che, vista la loro giovanissima età, si stanno affacciando sulla scena della "managerialità", si possono utilizzare i modelli, gli strumenti e le tecniche della creazione di valore?

Tale quesito è senz'altro degno di essere approfondito, in quanto introdurre nella Sanità concetti di razionale utilizzo delle risorse e di ottimale soddisfazione dei portatori di interessi rappresenta indubbiamente un tema rilevante e straordinariamente attuale.

Per dare alla domanda prima formulata una risposta corretta e non affrettata, senza cadere nella facile tentazione di impiegare acriticamente modelli già preconfezionati per altri settori, è utile mettere a fuoco il vero obiettivo strategico delle aziende sanitarie, ripercorrendo lo schema di analisi visto precedentemente per le imprese private. ${ }^{\mathrm{ii}}$

Così facendo si può rilevare che tra le imprese private e le aziende sanitarie esiste una sostanziale differenza: mentre per le imprese private l'interesse prioritario da soddisfare e di conseguenza l'obiettivo strategico da perseguire ha come riferimento l'azionista, per le aziende sanitarie in cima a tutti stanno invece gli interessi e i bisogni dell'utenza.

Da questa differenza derivano una serie di conseguenze sia nel significato che assume la creazione di valore sia nelle misure che possono rappresentarla.

1. Prima di tutto è evidente che la creazione di valore deve avere nella soddisfazione dei bisogni di salute degli utenti il suo principale punto di appoggio.

2. Secondariamente occorre rilevare che per le aziende sanitarie la differenza tra ricavi e costi perde una parte del suo significato (sintesi di efficienza e di efficacia) per ridursi a puro indicatore di efficienza. Ciò è da imputare al fatto che i ricavi delle aziende sanitarie

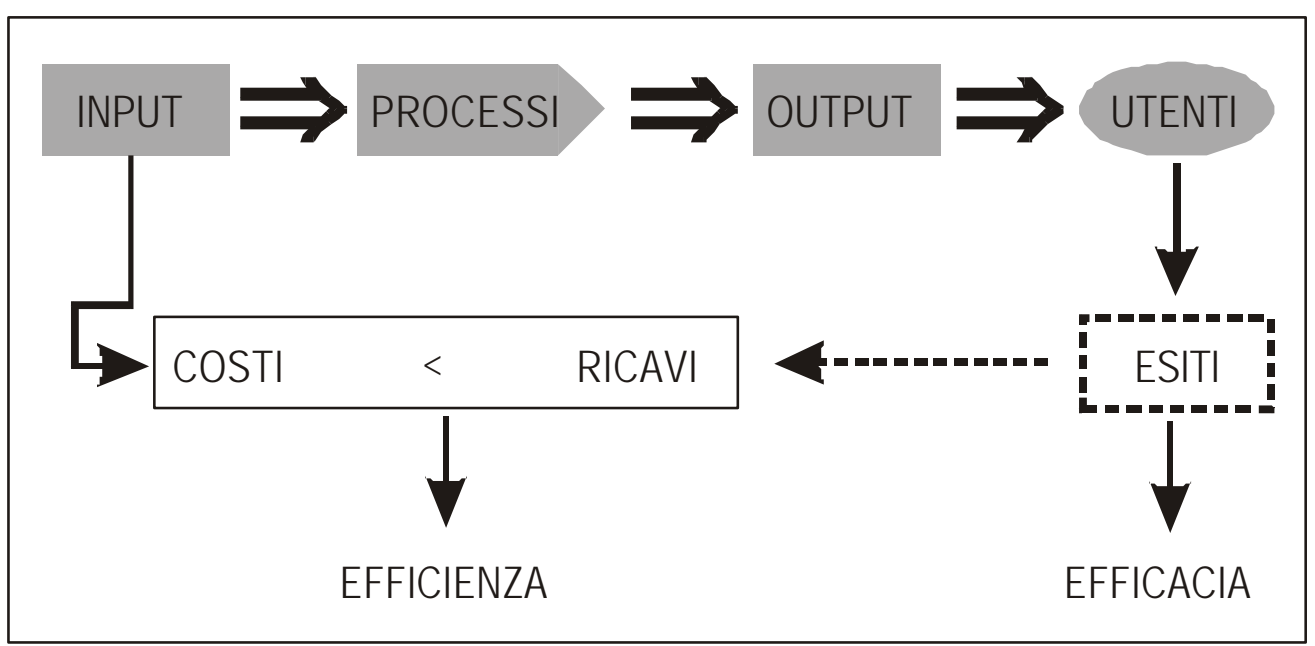

\section{Nota ii}

Anche le aziende sanitarie impiegano risorse di vario genere (materie prime, personale, risorse finanziarie) per produrre degli output (le prestazioni sanitarie) a fronte dei quali vi sono i bisogni di salute dell'utenza da soddisfare. Se questo processo è gestito al meglio le attese di salute

dell'utenza troveranno riscontro in esiti di salute (outcome) positivi. Il processo appena rappresentato può essere visto in termini di risvolti monetari: vi saranno spese per acquisire e quindi remunerare le risorse necessarie ( $i$ costi) ed entrate (i ricavi finanziamentil remunerazioni) concessi alle aziende sanitarie.

Figura 2

L'azienda sanitaria 


\section{Nota iii}

La creazione di valore in Sanità può essere assimilata, in un certo senso, a un rendimento del capitale investito. Quello che cambia è il metro di giudizio della bontà dell'investimento: non un rendimento in denaro ci si aspetta da tali aziende ma un rendimento in termini di risultati di salute ottenuti.

\section{Nota iv}

Così come il profitto o altri indicatori economici sono misure oggettive e quantificabili della creazione di valore delle imprese private. non sono una somma di prezzi di mercato bensì assumono la connotazione di tariffe o di trasferimenti di risorse: non sono quindi indicatori di soddisfazione dei bisogni degli utenti così come avviene per le imprese private.

Se il profitto fosse il primario obiettivo di un'azienda sanitaria ci si potrebbe trovare paradossalmente in una situazione in cui non sono tenuti in debita considerazione gli interessi prioritari dell'utenza: un'azienda sanitaria potrebbe infatti ottenere "brillanti" risultati economici riducendo il livello di servizio e la qualità delle prestazioni disattendendo di fatto la sua finalità e la sua missione.

3. Nonostante ciò, l'efficienza è comunque una condizione irrinunciabile della gestione e una prerogativa per la creazione di valore: un'azienda sanitaria non può soddisfare gli interessi dell'utenza se non è (anche) in grado di utilizzare in modo efficiente le risorse (scarse) a disposizione.

Come prima conclusione dei ragionamenti sin qui sviluppati possiamo pertanto ricavare i seguenti assunti da prendere come riferimenti di base per il modello di creazione di valore in Sanità:

? creare valore significa soddisfare gli interessi dell'utenza attraverso ottimali esiti di salute;

? la creazione di valore non può essere apprezzata da misure economico-monetarie quali il profitto (che per tali realtà rappresenta solo un indicatore di efficienza);

? la creazione di valore non può essere disgiunta dall'efficienza economica; quest'ultima peraltro, da obiettivo principale, diventa condizione strumentale della gestione. iii

Prima di addentrarci nello sviluppo del modello di creazione di valore riteniamo importante fare due ordini di riflessioni.

Il primo di carattere tecnico relativo alla misurazione dello stato di salute, il secondo di profilo più generale inerente i caratteri di innovatività del modello della crea- zione di valore.

Abbiamo individuato nello stato di salute il principale riferimento per poter apprezzare la capacità di creare valore di un'azienda sanitaria.

Da questo emerge l'importanza di approfondire meglio il concetto di stato di salute evidenziando le diverse variabili che, congiuntamente all'operato delle aziende sanitarie, concorrono a determinarlo. Lo stato di salute di una popolazione è infatti il risultato dell'azione di diversi fattori di varia natura (Figura 3):

? le scelte di politica sanitaria, per citare forse quella a maggiore impatto pensiamo al livello di risorse dedicate alla sanità;

? le caratteristiche della popolazione, sotto il profilo demografico ed epidemiologico;

? gli stili di vita, con riferimento alle abitudini alimentari, o alla diffusione del fumo o del consumo di alcool e così via;

? i fattori ambientali quali le condizioni climatiche, l'inquinamento atmosferico ecc.

Tutti questi elementi, sostanzialmente esogeni rispetto alle aziende sanitarie, hanno un riflesso congiunto sullo stato di salute di una popolazione unendosi agli effetti che produce la gestione delle stesse aziende.

Tali relazioni sono da tenere in stretta considerazione specialmente quando si devono definire indicatori e misure dello stato di salute per poter valutare la creazione di valore delle aziende sanitarie. In particolare è indispensabile trovare misure che esprimano la creazione di valore in modo oggettivo, o il meno soggettivo possibile. ${ }^{\text {iv }}$ Se così non fosse si rischierebbe di effettuare valutazioni non corrette utilizzando, ad esempio, indicatori che misurano il risultato complessivo dell'azione di tutte le variabili prima indicate.

Anche a tale proposito possono essere fissati alcuni punti fermi da seguire.

? Poiché lo stato di salute è funzione di diverse variabili, occorre identificare misure che

\section{Figura 3}

Lo stato di salute

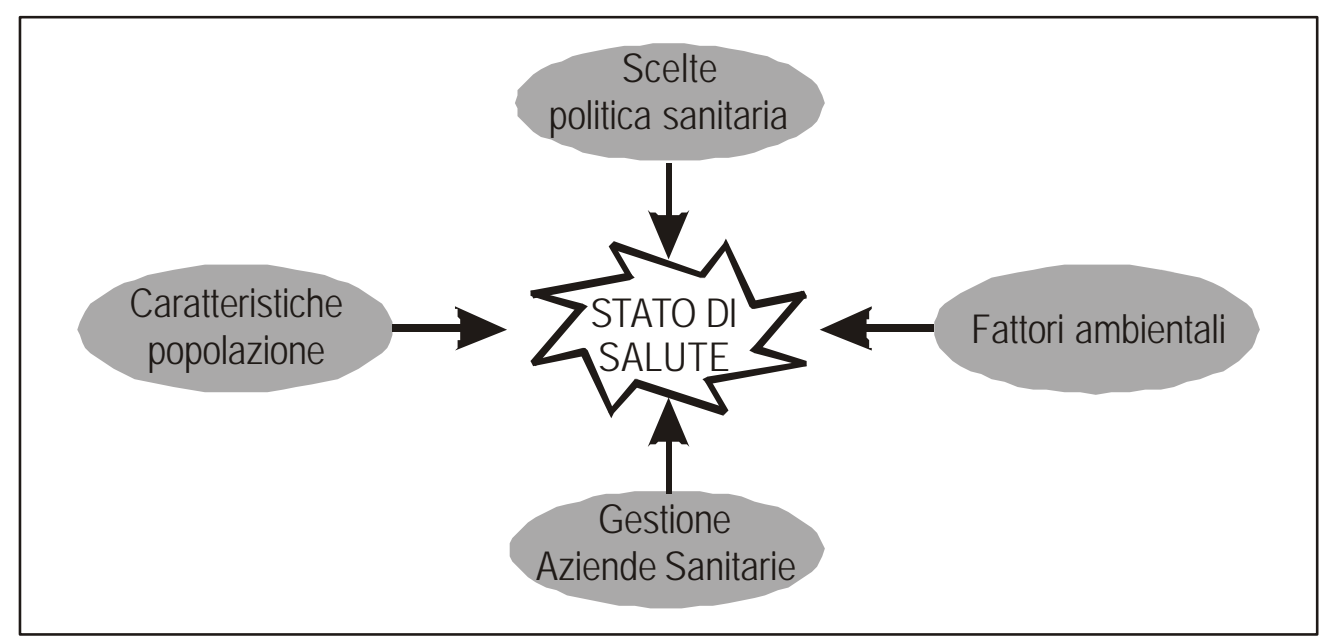


permettano di apprezzare il contributo relativo offerto dalle aziende sanitarie allo stato di salute complessivo, contributo che d'ora in avanti chiameremo health value added (HVA). ? Tale contributo difficilmente può essere misurato da indicatori quali i tassi di mortalità o di incidenza di nuove patologie poiché rappresentano misure:

? di lungo periodo, che risentono solo in un tempo relativamente ampio dell'effetto delle variabili da cui dipende lo stato di salute;

? di sintesi, ovvero che colgono l'effetto congiunto di tutti i fattori prima indicati.

? Alla luce di quanto esposto nei punti precedenti, emerge in modo netto la necessità di orientarsi verso indicatori di breve periodo che misurano gli effetti dell'azione delle aziende sanitarie sullo stato di salute.

? Nel caso in cui l'individuazione risulti difficile, o rappresenti una modalità di misurazione non completa, è necessario integrare tale valutazione con misurazioni indirette della creazione di valore. ${ }^{\mathrm{V}}$

La seconda riflessione che riteniamo di dover fare è relativa ai tratti di innovatività che il modello della creazione di valore presenta rispetto agli strumenti di management tradizionalmente utilizzati nelle aziende sanitarie.

Queste ultime hanno infatti interpretato il management principalmente come strumento per governare e ridurre i costi. In realtà gli obiettivi di efficienza, validi in generale per qualunque impresa e, a maggior ragione, per aziende come quelle sanitarie sottoposte a ristretti vincoli di risorse disponibili, non rappresentano la vera finalità di un'azienda sanitaria.

Contrariamente a tale logica, il comune denominatore delle applicazioni reali è stato invece la focalizzazione sul contenimento dei costi con risultati, sotto questo profilo, peraltro abbastanza modesti.

A completare tale quadro è da citare inoltre anche il difficile innesto che gli strumenti di management hanno avuto sull'operatività della componente medico-sanitaria.

Meccanismi e procedure di budget e di controllo di gestione hanno infatti incontrato notevole difficoltà a farsi strada nel mondo medico, proprio in virtù del malinteso di fondo sulle loro finalità.

In tale ottica si ritiene che il modello della creazione di valore permetta di riequilibrare la prospettiva dei sistemi di controllo, ridando centralità alle variabili tradizionalmente trascurate quali l'efficacia e la qualità dei processi diagnostico-terapeutici e di conseguenza anche alla componente medica.

\section{Il percorso di creazione di valore}

Aver posto il concetto di creazione di valore per l'utenza come finalità di un modello di management rischia di essere una dichiarazione di principio che, pur condivisibile a priori, può risultare scarsamente utile se non tradotta e articolata concretamente.

Per dare corpo a tale modello e tradurlo in

\section{Nota $\mathbf{v}$}

Ad esempio deducendo la capacità di creare valore dalle variabili gestionali che ne costituiscono le premesse. Tale aspetto sarà meglio chiarito nel prosieguo del lavoro.

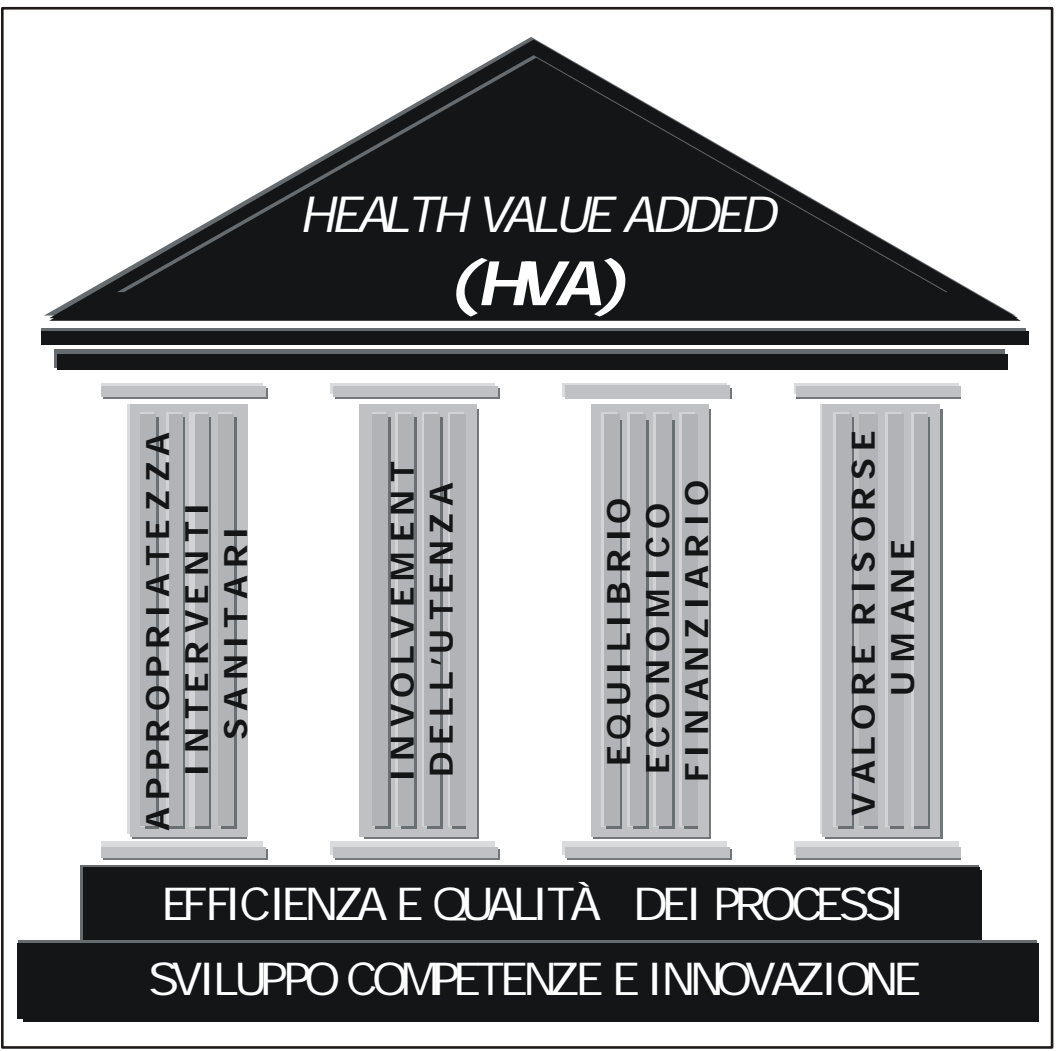

Figura 4

Il modello di creazione di valore per le aziende sanitarie 
concreti strumenti di supporto alla gestione, è sfatto e con alti livelli di motivazione (valore necessario articolare il concetto di valore per risorse umane);

l'utenza, identificando il percorso e le varia- 4. il coinvolgimento dell'utenza nel procesbili da cui questo dipende.

Un utile ragionamento da condurre a tal fine può essere l'individuazione delle variabili partendo dalla definizione delle "qualità" che una azienda sanitaria dovrebbe, in teoria, avere per poter creare valore.

Queste sono sostanzialmente riassumibili in cinque categorie:

es la qualità sanitaria;

es la qualità manageriale e organizzativa;

es la qualità del personale;

es la qualità percepita dall'utenza, intesa come capacità di comunicare e far percepire la qualità sanitaria;

es la qualità economica della gestione.

Declinando in dettaglio tali requisiti, attraverso la definizione di un sistema di variabili, è possibile concretizzare il nostro modello.

Per raffigurare efficacemente il modello può essere utilizzata una rappresentazione a forma di tempio (Figura 4), il cui frontone indichi la creazione di valore e le parti che lo sorreggono esprimano invece le variabili che ne costituiscono le premesse e le condizioni di raggiungimento.

Così come il frontone necessita di colonne e basamenti per poter stare in piedi, allo stesso modo, per creare valore, sono necessari premesse e presupposti derivati dalle qualità prima indicate.

Facciamo in particolare riferimento ai seguenti punti:

1. l'esistenza di processi e attività efficienti $e$ di qualità (sia di carattere medico-sanitario che amministrativo-gestionale);

2. l'appropriatezza degli interventi sanitari, intesa come la prerogativa di "fare solo quello che serve", coerentemente con l'obiettivo di salute che si vuole raggiungere;

3. la disponibilità di personale valido, soddiso di erogazione delle cure con appropriata comunicazione (involvement dell'utenza);

5. al fine di garantire le predette condizioni è necessario sviluppare e preservare nel tempo le competenze distintive aziendali attraverso la ricerca e l'innovazione;

6. infine, non certo in ordine di importanza, l'equilibrio economico-finanziario da intendersi come la capacità di "farsi bastare le risorse a disposizione".

Questi sei elementi, di cui uno rappresenta un vincolo (equilibrio economico finanziario) e gli altri invece obiettivi di tipo intermedio, costituiscono le condizioni necessarie affinché un'azienda sanitaria possa creare valore per la propria utenza.

Possiamo bene intuire l'importanza che gli elementi citati e la loro "robustezza" hanno al fine di creare valore, così come è facile capire le conseguenze che si avrebbero nel caso in cui il tempio mancasse di alcune colonne, avesse colonne non sufficientemente solide per sostenere l'architrave o infine poggiasse su una base fragile.

Gli esempi di fragile o imperfetta "costituzione" sono molteplici e tutti, purtroppo, riscontrabili più o meno frequentemente nella realtà. Si possono citare infatti casi di aziende sanitarie o strutture che:

es erogano efficienti prestazioni di qualità, in equilibrio economico, ma che non rispettano i criteri di appropriatezza delle cure ("fanno cose ben fatte ma inutili");

2s pur presentando tutti i requisiti non riescono ad alimentare nel tempo le proprie competenze con l'innovazione e la ricerca necessari;

2s annoverano tra le loro fila validi professionisti, soddisfatti e motivati ma con carenze sotto il profilo dell'efficienza e quindi in disequilibrio economico.

Figura 5

Il percorso di creazione di valore

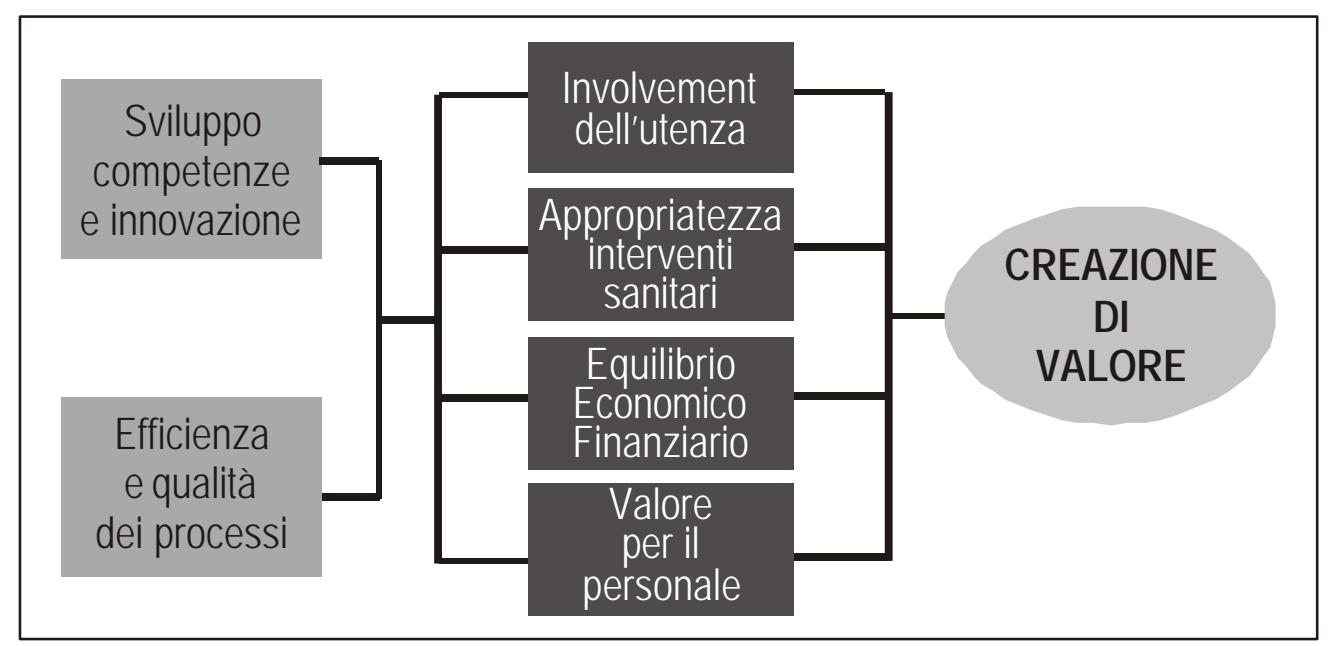


Questi, insieme a molti altri, sono tutti casi di aziende che nel breve o nel medio periodo sperimenteranno la difficoltà di soddisfare in modo ottimale le esigenze di salute dei propri assistiti e non saranno quindi in grado di creare valore.

Quanto sin qui esposto illustra come il percorso di creazione di valore (Figura 5) sia articolato e composito e necessiti, per poter essere portato a compimento, di molteplici elementi. La creazione di valore è infatti il prodotto finale di un "gioco di squadra" che coinvolge una serie ampia di variabili, il cui comportamento deve essere coordinato e bilanciato in modo sinergico. In particolare le variabili del modello sono articolate tra loro secondo un duplice criterio:

? causale, per cui si individuano obiettivi finali di stato di salute (valore per l'utenza), e obiettivi intermedi (o strumentali) finalizzati al raggiungimento dei primi, e quindi logicamente collegati ad essi da una relazione di causa/effetto che il management necessariamente deve presupporre; ${ }^{\text {i }}$

? temporale, nel senso che il perseguimento e la realizzazione della creazione di valore richiede un bilanciamento tra azioni i cui effetti si concretizzeranno in un arco temporale di breve termine, e obiettivi di medio-lungo termine finalizzati, a preparare le condizioni per garantire nel lungo termine la capacità di creare valore. Appartengono a questa categoria gli obiettivi legati a progetti di innovazione e sviluppo delle competenze, la ricerca, la formazione delle risorse, i cui effetti sullo stato di salute della popolazione non si avranno nell'immediato, ma in un orizzonte temporale più ampio.

\section{I sistemi di programmazione e controllo al} supporto della creazione di valore

Il modello del valore così articolato costituisce una struttura concettuale "statica" che deve essere tradotta di strumenti e meccanismi di supporto alla gestione.

A tale scopo possono essere proficuamente impiegati i tradizionali "attrezzi" della programmazione e controllo, rivisti e ritarati in ottica di creazione di valore.

Tale strumentazione, lo ricordiamo, si basa su una logica molto semplice: occorre programmare (stabilire prima a quali risultati si intende arrivare) e controllare la gestione (verificare poi se i risultati che si vanno realizzando sono in linea con quelli attesi).

In particolare è necessario:

? prima, fissare gli obiettivi e programmare gli impegni e le attività, in modo da rendersi conto in anticipo dei possibili problemi che si potranno incontrare e studiare quindi le contromisure e le soluzioni;

? successivamente, nel corso della gestione, accertare se "le cose vanno secondo le attese", cioè se la gestione si sta svolgendo in modo corrispondente ai programmi, per rendersi conto tempestivamente dell'esigenza di adottare provvedimenti correttivi appropriati;

? infine, verificare a consuntivo se i risultati effettivi hanno corrisposto a quelli programmati, in modo da "tarare meglio il tiro" nell'elaborazione dei successivi programmi.

Lo sforzo da compiere rispetto a questo modello generale è quello di adeguare tale formula alle logiche del valore, riempiendo di contenuti specifici strumenti quali la definizione degli obiettivi strategici, il budget, il reporting.

In tale prospettiva, un modello di pianificazione e controllo basato sulla logica di creazione di valore potrebbe articolarsi nel modo seguente.

\section{La definizione degli obiettivi strategici}

La prima fase è quella relativa alla definizione degli obiettivi strategici che l'azienda intende perseguire. Tali obiettivi costituisco-

\section{Nota vi}

È evidente la difficoltà che deriva da questa attività di identificazione dei rapporti causali, ma è altrettanto vero che senza di essa l'attività del management rischia di limitarsi ad una dichiarazione di intenti non supportata da azioni operative concrete.

\begin{tabular}{|l||l|}
\hline $\begin{array}{l}\text { Quali risultati si intendono ottenere in termini di } \\
\text { miglioramento dello stato di salute dei pazienti trattati e della } \\
\text { popolazione assistita in generale? }\end{array}$ & Creazione di valore per l'utenza \\
\hline $\begin{array}{l}\text { Quali attività e processi si intendono realizzare per } \\
\text { raggiungere gli obiettivi di salute e di prevenzione } \\
\text { individuati? }\end{array}$ & $\begin{array}{l}\text { Appropriatezza interventi sanitari } \\
\text { Processi }\end{array}$ \\
\hline \hline $\begin{array}{l}\text { Quali azioni occorre attivare per qualificare e motivare il } \\
\text { capitale umano dell'azienda? }\end{array}$ & Valore risorse umane \\
\hline $\begin{array}{l}\text { Quali livelli di servizio e di qualità si vogliono comunicare e } \\
\text { far percepire all'utenza? }\end{array}$ & Involvement dell'utenza \\
\hline $\begin{array}{l}\text { Quali competenze e capacità sono necessarie per } \\
\text { conseguire tali risultati e quali azioni si intendono attivare } \\
\text { per preservarle e alimentarle? }\end{array}$ & Innovazione e sviluppo competenze \\
\hline $\begin{array}{l}\text { Quale sarà l'impegno in termini di risorse economiche e } \\
\text { finanziarie richiesto dagli obiettivi definiti? }\end{array}$ & Equilibrio economico-finanziario \\
\hline
\end{tabular}


no la declinazione della mission dell'azienda e devono essere articolati secondo le variabili prima indicate. La definizione degli obiettivi strategici consente di rispondere ai seguenti quesiti di fondo. (Tabella 1)

Per ognuno dei temi strategici prima indicati è necessario individuare obiettivi e indicatori di misurazione ed esplicitare azioni e attività da svolgere.

Tale fase deve auspicabilmente vedere il coinvolgimento di tutto il top management dell'azienda ed essere strutturata in un processo di analisi, definizione e articolazione accurato e condiviso.

La programmazione della gestione: il programma (Figura 6)

Gli obiettivi strategici prima individuati costituiscono un riferimento programmatico che l'azienda deve seguire nel medio-lungo periodo. Nel breve periodo, tipicamente l'anno, tali riferimenti vanno ulteriormente declinati in micro-obiettivi, corredati di azioni e risorse necessarie per il loro raggiungimento.

La programmazione segue una logica di sviluppo simile a quella utilizzata per sviluppare gli obiettivi strategici. In dettaglio questa fase si configura nella redazione di specifici piani e programmi per ogni focus di gestione:

1. il piano degli obiettivi di salute;

2. il piano delle attività e dei processi;

3. il piano di sviluppo delle competenze e del capitale umano;

4. il budget economico-finanziario.

In sintesi, il piano degli obiettivi di salute identifica il livello di salute da assicurare, attraverso uno studio preliminare della domanda e della concorrenza e una valutazione delle priorità di intervento.

Il piano delle attività permette di definire le attività da svolgere per raggiungere il livello di salute programmato. Si esplicita in un programma delle attività e delle prestazioni da effettuare con identificazione delle risorse necessarie per portare a compimento tali azioni.

Il piano della qualità percepita consente di individuare le attività relative alla comunicazione e il livello obiettivo di qualità percepita che si intendono ottenere nel periodo di budget.

Il piano sviluppo delle competenze e del capitale umano identifica obiettivi, azioni e costi necessari a garantire la motivazione del capitale umano e a portare le competenze dell'azienda verso i livelli che le consentano di raggiungere e mantenere nel tempo l'efficacia e l'efficienza programmate.

Infine il budget economico finanziario consente di tradurre in termini economico-finanziari le scelte fatte in sede di definizione degli obiettivi e delle azioni da svolgere, e di sottoporre quindi le fasi di programmazione alla prova dell'equilibrio economico-finanziario.

Una schematizzazione delle fasi della programmazione è indicata di seguito.

Il controllo della gestione: $i$ sistemi di balanced scorecard (Figura 7)

L'ultima fase del processo è quella del controllo della gestione attraverso il reporting.

Tale fase, coerentemente con il modello sin qui definito, dovrebbe consentire di individuare se e in che misura l'azienda sta creando valore.

Il modello di reporting deve pertanto strutturarsi come un cruscotto di informazioni ad ampio spettro su tutte le variabili da cui dipende la creazione di valore.

Modelli di questo tipo si stanno sviluppando con grande intensità nel mondo delle imprese private e prendono il nome di sistemi di balanced scorecard.

I sistemi di balanced scorecard consentono di controllare al meglio tutte le variabili che costituiscono i presupposti della creazione di valore:
Figura 6

La programmazione della gestione

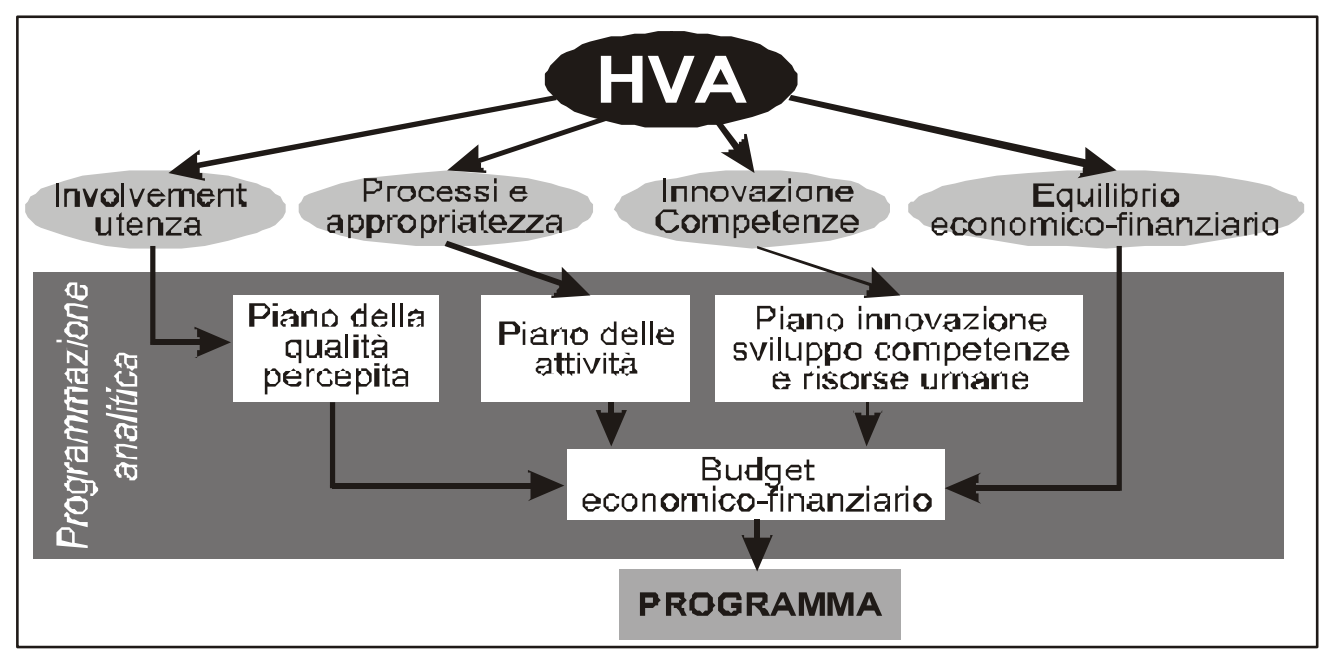




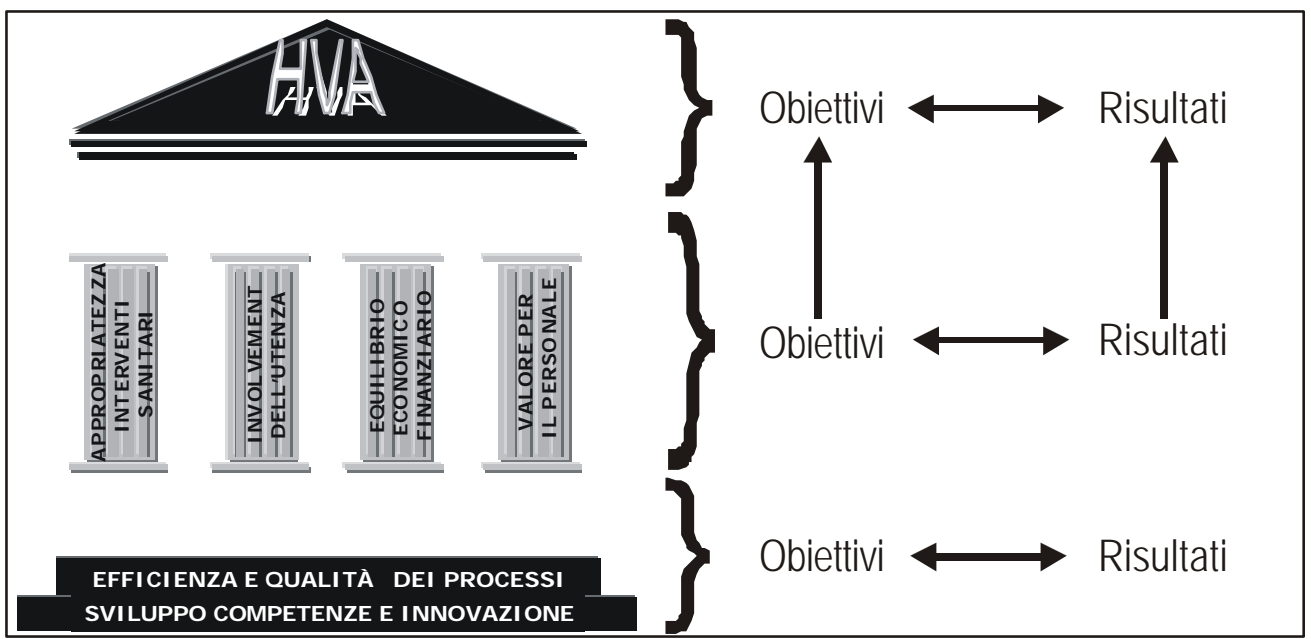

1. gli obiettivi intermedi o secondari relativi obiettivi di salute?

ai processi, ai loro costi e qualità, alle azioni 3. quali azioni di sviluppo delle competenze sulle risorse umane, ai progetti di innovazio- e delle risorse umane sono state intraprese rine, ricerca e sviluppo delle competenze; spetto agli obiettivi programmati?

2. i vincoli di equilibrio di gestione sotto il profilo economico-finanziario.

Il balanced scorecard permette di evidenziare i risultati della gestione, rispondendo alle seguenti domande:

1. quali risultati di stato di salute sono stati conseguiti?

2. quali attività sono state effettuate e quali costi sono stati sostenuti per raggiungere gli
4. quale impatto i primi tre punti hanno avuto sotto il profilo economico-finanziario del-

Tutte le informazioni relative ai seguenti punti possono essere fornite in un quadro sintetico che evidenzi, in modo efficace e visivo, l'andamento della gestione come in un cruscotto di controllo.

Nella Figura 8 è indicato un esempio di la gestione?
Figura 7

La logica dei sistemi dibalanced scorecard

\begin{tabular}{|c|c|c|c|}
\hline FOCUS & VARIABILE & INDICATORE & TARGET \\
\hline Involvement dell'utenza & $\begin{array}{l}\text { Qualità percepita del } \\
\text { servizio } \\
\text { Qualità percepita delle } \\
\text { strutture } \\
\text { Reclami }\end{array}$ & $\begin{array}{l}\text { Disponibilità, Cortesia, } \\
\text { Informazioni ricevute } \\
\text { Comfort alberghiero, } \\
\text { Segnaletica interna } \\
\text { Numero } \\
\text { Tipologia }\end{array}$ & \\
\hline Valore per il personale & $\begin{array}{l}\text { Motivazione } \\
\text { Clima } \\
\text { Senso appartenenza }\end{array}$ & \begin{tabular}{|l} 
Grado di raggiungimento \\
obiettivi \\
Assenteismo \\
Giorni di malattia \\
Richieste di trasferimento \\
interno \\
Anzianità media di \\
servizio \\
Turnover
\end{tabular} & \\
\hline FOCUS & VARIABILE & INDICATORE & TARGET \\
\hline $\begin{array}{l}\text { Equilibrio economico- } \\
\text { finanziario }\end{array}$ & $\begin{array}{l}\text { Economico } \\
\text { Finanziario }\end{array}$ & $\begin{array}{l}\text { Reddito operativo } \\
\text { ROI } \\
\text { Incidenza costo risorse } \\
\text { su ricavi } \\
\text { Spesa per livello di } \\
\text { assistenza } \\
\% \text { utilizzo anticipazione } \\
\text { Giacenza media fornitori } \\
\text { e scorte }\end{array}$ & \\
\hline
\end{tabular}


Figura 8

Il balanced scorecard

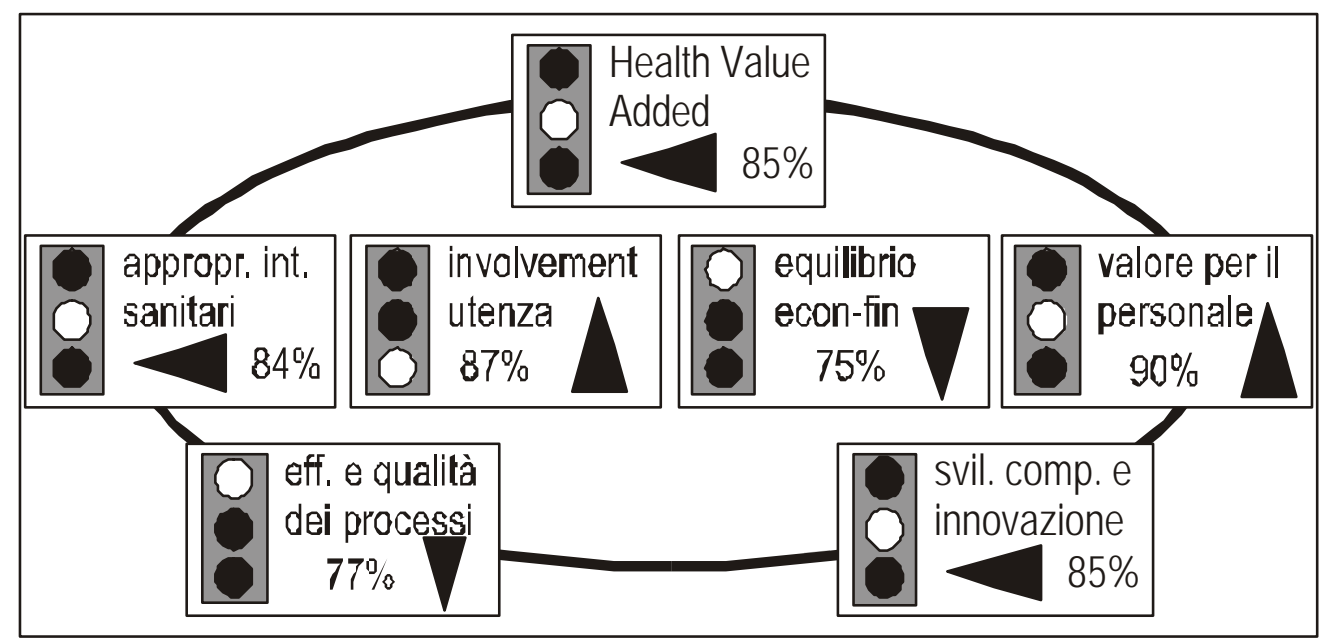

balanced scorecard espresso sotto forma segnaletica di semaforo.

Con riferimento ad ogni focus della gestione (processi, involvement utenza, ecc..) è indicata la percentuale di raggiungimento degli obiettivi confrontando, per ogni variabile chiave, i risultati effettivi con quelli attesi.

Ovviamente il balanced scorecard deve permettere fasi di approfondimento più dettagliate attraverso lo studio dei dati analitici che sottostanno agli indicatori di sintesi.

La fase di analisi deve consentire di mettere a fuoco per ogni variabile:

? l'andamento effettivo rispetto a quello programmato, tramite l'evidenziazione di eventuali scostamenti;

? le cause che hanno portato a non rispettare gli impegni;

? le azioni correttive da intraprendere. (Tabella 2)

Questa analisi è possibile in quanto, nella fase di costruzione del modello di creazione di valore, si è provveduto a individuare, per ognuno dei focus della gestione, le variabili, gli indicatori di controllo e $\mathrm{i}$ target da raggiungere.

A titolo di esempio, precedentemente, sono stati indicati variabili e indicatori relativi al focus involvement dell'utenza, valore per il personale ed equilibrio economico-finanziario. (Tabella 2)

È opportuno sottolineare che quella presentata è solo una delle possibili configurazioni di dettaglio delle variabili e degli indicatori. Ogni azienda dovrà identificare la propria, in funzione di alcuni fattori quali le caratteristiche del proprio bacino di utenza, della concorrenza e le proprie peculiarità strutturali.

\section{CONCLUSIONI}

Come conclusione di questo scritto possiamo affermare che il modello della creazione di valore può risultare una valida proposta per innovare i sistemi di controllo delle aziende sanitarie e consentire di superare l'impasse in cui si trovano molte applicazioni di management sanitario.

L'ipotesi presentata ha il pregio di voler riportare al centro dei modelli di controllo l'efficacia e i bisogni dell'utenza.

Questa caratteristica è fondamentale anche per avvicinare all'uso di tali strumenti la componente medica che, pur dovendo rivestire un ruolo primario, ha finito per essere, in molti casi, la grande esclusa.

La marcia di avvicinamento tra managerialità e classe medica deve peraltro registrare anche qualche passo da parte di quest'ultima. In particolare, sarebbe necessario modificare l'atteggiamento di riluttanza che i medici hanno avuto e hanno nei confronti degli strumenti di management; questo comportamento, anche se in parte giustificato dai problemi a cui abbiamo prima accennato, è a volte un sintomo della scarsa disponibilità ad accettare sistemi di valutazione e misurazione dell'attività svolta.

Solo se questi passi saranno compiuti, i pregi che il modello di creazione di valore dimostra potenzialmente di poter avere, potranno "passare" dalle pagine della teoria alla realtà. 